\title{
Discrete Characterizations of Exponential Dichotomy for Evolution Families
}

\author{
PETRE PREDA, ALIN POGAN AND CIPRIAN PREDA
}

\begin{abstract}
We present some characterizations of exponential dichotomy using a discrete argument. The results obtained generalize to the case of exponential dichotomy some theorems proved by Littman, Rolewicz and Zabczyk.
\end{abstract}

\section{INTRODUCTION}

One of the most remarkable results in the theory of stability for a strongly continuous semigroup of linear operators has been obtained by Datko [2] in 1970; it states that the semigroup $T=\{T(t)\}_{t \geq 0}$ is uniformly exponentially stable if and only if, for each vector $x$ from the Banach space $X$, the application $t \mapsto\|T(t) x\|$ lies in $L^{2}\left(\mathbf{R}_{+}\right)$. Later, A. Pazy (see for instance [10]) showed that the result remains true even if we replace $L^{2}\left(\mathbf{R}_{+}\right)$with $L^{p}\left(\mathbf{R}_{+}\right)$, where $p \in[1, \infty)$. In 1973, R. Datko [3] generalized the results above as follows.

Theorem 1.1. An evolutionary process $\mathcal{U}=\{U(t, s)\}_{t \geq s \geq 0}$ with exponential growth is uniformly exponentially stable if and only if there is $p \in[1, \infty)$ such that

$$
\sup _{s \geq 0} \int_{s}^{\infty}\|U(t, s) x\|^{p} d t<\infty \quad(x \in X) .
$$

The result provided by Theorem 1.1 was extended to dichotomy by P. Preda and M. Megan [14] in 1985. The same result was generalized in 1986 by S. Rolewicz [16] in the following way.

2000 Mathematics Subject Classification. Primary 34D09; Secondary 34D05, 39A12, 47D06.

Key words and phrases. Evolution families, exponential dichotomy. 
Theorem 1.2. Let $\phi: \mathbf{R}_{+} \rightarrow \mathbf{R}_{+}$be a continuous, nondecreasing function with $\phi(0)=0$ and $\phi(u)>0$ for each positive $u$, and $\mathcal{U}=$ $\{U(t, s)\}_{t \geq s \geq 0}$ an evolutionary process on $X$ with exponential growth. If

$$
\sup _{s \geq 0} \int_{s}^{\infty} \phi(\|U(t, s) x\|) d t<\infty \quad(x \in X),
$$

then $\mathcal{U}$ is uniformly exponentially stable.

We note here the result obtained independently by Littman [6] in 1989 , in the case of $C_{0}$-semigroups but without the assumption of continuity of $\phi$.

Results of this type, for the case of $C_{0}$-semigroups were provided by I. Zabczyk [17] in 1974, with the additional requirement that the function $\phi$ is also convex, as can be seen below:

Theorem 1.3. For every $C_{0}$-semigroup $T=\{T(t)\}_{t \geq 0}$ the following statements are equivalent:

(i) $T$ is exponentially stable;

(ii) there is a convex increasing function $\phi: \mathbf{R}_{+} \rightarrow \mathbf{R}_{+}$vanishing at 0 and for every $x \in X$ there is $\alpha(x)>0$ such that

$$
\int_{0}^{\infty} \phi(\alpha(x)\|T(t) x\|) d t<\infty \quad(x \in X)
$$

(iii) there is a convex increasing function $\varphi: \mathbf{R}_{+} \rightarrow \mathbf{R}_{+}$with $\varphi(0)=0$ and for every $x \in X$ there is $\alpha(x)>0$ such that

$$
\sum_{n=0}^{\infty} \varphi(\alpha(x)\|T(n) x\|)<\infty \quad(x \in X) .
$$

Also, more recently, an unified treatment was presented by J. M. A. M. Neerven [8] in terms of Banach functions spaces.

The aim of this paper is to extend the preceding results to the case of exponential dichotomy using a discrete time argument.

\section{Preliminaries}

In the beginning we will fix some standard notation. We denote by $\mathcal{A}$ the set of all non-decreasing functions $a: \mathbf{R}_{+} \rightarrow \mathbf{R}_{+}$with the property that $a(t)>0$ for all $t>0$. In what follows we will put $X$ for a Banach space and $\mathcal{B}(X)$ the Banach algebra of all linear and bounded operators acting on $X$. 

Remark 2.0. If $a \in \mathcal{A}$ and $A: \mathbf{R}_{+} \rightarrow \mathbf{R}_{+}, A(u)=\int_{0}^{u} a(s) d s$, then
$A \in \mathcal{A}$ and $A$ is a continuous convex bijection.

Definition 2.1. A family of bounded linear operators acting on $X$ and denoted by $\mathcal{U}=\{U(t, s)\}_{t \geq s \geq 0}$ is called an evolution family if the following properties hold:

$\left.e_{1}\right) U(t, t)=I$ (the identity operator on $X$ ), for all $t \geq 0$;

$\left.e_{2}\right) U(t, s)=U(t, r) U(r, s)$, for all $t \geq r \geq s \geq 0$;

$\left.e_{3}\right)$ there exist $M, w>0$ such that

$$
\|U(t, s)\| \leq M e^{w(t-s)} \quad, \quad \text { for all } \quad t \geq s \geq 0 .
$$

In order to deal with the dichotomy property we give the following:

Definition 2.2. A function $P: \mathbf{R}_{+} \rightarrow \mathcal{B}(X)$ is said to be a dichotomy projection family if

$\left.p_{1}\right) P^{2}(t)=P(t)$, for all $t \geq 0$;

$\left.p_{2}\right) P(\cdot) x$ is a bounded function, for all $x \in X$.

We also denote by $Q(t)=I-P(t), t \geq 0$.

Definition 2.3. An evolution family $\mathcal{U}$ is said to be uniformly exponentially dichotomic (u.e.d.) if there exists $P$ a dichotomy projection family and two constants $N, \nu>0$ such that the following conditions hold:

$\left.d_{1}\right) P(t) U(t, s)=U(t, s) P(s)$ for all $t \geq s \geq 0$;

$\left.d_{2}\right) U(t, s): \operatorname{Ker} P(s) \rightarrow \operatorname{KerP}(t)$ is an isomorphism for all $t \geq$ $s \geq 0$;

$\left.d_{3}\right)\|U(t, s) x\| \leq N e^{-\nu(t-s)}\|x\|$, for all $t \geq s \geq 0$, and all $x \in$ $\operatorname{ImP}(s)$;

$\left.d_{4}\right)\|U(t, s) x\| \geq \frac{1}{N} e^{\nu(t-s)}\|x\|$, for all $t \geq s \geq 0$, and all $x \in$ $\operatorname{KerP}(s)$.

In what follows we will consider an evolution family $\mathcal{U}$ for which there is a dichotomy projection family $P$ such that the properties $d_{1}$ ) and $\left.d_{2}\right)$ hold. In this case we will denote by

$$
U_{1}(t, s)=U(t, s)\left|\operatorname{ImP}(s) \quad, \quad U_{2}(t, s)=U(t, s)\right| \operatorname{KerP}(s) .
$$

Even if all the conditions $\left.\left.e_{1}\right), e_{2}\right), e_{3}$ ) and $\left.d_{1}\right), d_{2}$ ) are satisfied, it does not follows that $U_{2}^{-1}$ has exponential growth, as the following example shows. 
Example 2.4. Let $X=\mathbf{R}, U(t, s)=e^{-\left(t^{2}-s^{2}\right)}, P(t)=0$. Then $U_{2}^{-1}(t, s)=e^{t^{2}-s^{2}}$, for all $t \geq s \geq 0$ and hence $U_{2}^{-1}$ does not have exponential growth.

Remark 2.5. The evolution family $\mathcal{U}$ is u.e.d. if and only if there exist the constants $N_{1}, N_{2}, \nu_{1}, \nu_{2}>0$ such that, for all $t \geq s \geq 0$,

$$
\left\|U_{1}(t, s)\right\| \leq N_{1} e^{-\nu_{1}(t-s)} \quad \text { and } \quad\left\|U_{2}^{-1}(t, s)\right\| \leq N_{2} e^{-\nu_{2}(t-s)} .
$$

Lemma 2.6. Let $g:\left\{(t, s) \in \mathbf{R}^{2}: t \geq s \geq 0\right\} \rightarrow \mathbf{R}_{+}$. If $g$ satisfy the conditions

i) $g(t, s) \leq g(t, r) g(r, s) \quad$, for all $t \geq r \geq s \geq 0$;

ii) $\sup _{0 \leq t_{0} \leq t \leq t_{0}+1} g\left(t, t_{0}\right)<\infty$;

iii) there exists $h: \mathbf{N} \rightarrow \mathbf{R}_{+}$with $\lim _{n \rightarrow \infty} h(n)=0$ such that

$$
g\left(m+n_{0}, n_{0}\right) \leq h(m) \quad\left(m, n_{0} \in \mathbf{N}\right),
$$

then there exist $N, \nu>0$ such that

$$
g\left(t, t_{0}\right) \leq N e^{-\nu\left(t-t_{0}\right)} \quad\left(t \geq t_{0} \geq 0\right) .
$$

Proof. Let $a=\sup _{0 \leq t_{0} \leq t \leq t_{0}+1} g\left(t, t_{0}\right), m_{0}=\min \left\{m \in \mathbf{N}: h(m) \leq \frac{1}{e}\right\}$.

Conditions i) and ii) imply that $\sup _{0 \leq t_{0} \leq t \leq t_{0}+m_{0}} g\left(t, t_{0}\right) \leq a^{m_{0}}$. Fix $t, t_{0} \geq 0$ with $t \geq t_{0}+2 m_{0}, m=\left[\frac{t}{m_{0}}\right], n=\left[\frac{t_{0}}{m_{0}}\right]$, where $[\mathrm{s}]$ denotes the largest integer less or equal than $s \in \mathbf{R}$. It follows that

$$
m m_{0} \leq t<(m+1) m_{0}, n m_{0} \leq t_{0}<(n+1) m_{0}, m \geq n+2,
$$

and

$$
\begin{aligned}
g\left(t, t_{0}\right) & \leq g\left(t, m m_{0}\right) g\left(m n_{0},(n+1) n_{0}\right) g\left((n+1) n_{0}, t_{0}\right) \\
& \leq a^{m_{0}} \prod_{k=n+2}^{m} g\left(k m_{0},(k-1) m_{0}\right) a^{m_{0}}=a^{2 m_{0}} \prod_{k=n+2}^{m} h\left(m_{0}\right) \\
& \leq a^{2 m_{0}} \prod_{k=n+2}^{m} e^{-1}=a^{2 m_{0}} e^{-(m-n-1)} \leq a^{2 m_{0}} e^{2-\frac{t-t_{0}}{m_{0}}}
\end{aligned}
$$


If $t_{0} \leq t \leq t_{0}+2 m_{0}$, then it follows easily that

$$
g\left(t, t_{0}\right) \leq a^{2 m_{0}} \leq a^{2 m_{0}} e^{2-\frac{t-t_{0}}{m_{0}}},
$$

and hence that

$$
\begin{gathered}
g\left(t, t_{0}\right) \leq N e^{-\nu\left(t-t_{0}\right)} \quad, \quad \text { for all } t \geq t_{0} \geq 0 \quad, \quad \text { where } \\
N=e^{2} a^{2 m_{0}} \quad, \quad \nu=\frac{1}{m_{0}} . \quad \square
\end{gathered}
$$

Lemma 2.7. If $a \in \mathcal{A}, \alpha: \mathbf{N}^{2} \rightarrow \mathbf{R}_{+}, v>0$ satisfy the following conditions:

i) $\sup \{\alpha(n, m): m, n \in \mathbf{N}, n \leq k\}<\infty$;

ii) there exists $C>0$ such that $\sum_{j=0}^{n} a\left(\frac{1}{e^{v j}} \alpha(n, m)\right) \leq C$, for all $m, n \in \mathbf{N}$, then $\sup _{m, n \in \mathbf{N}} \alpha(n, m)<\infty$. Proof. Assume towards a contradiction that $\sup _{m, n \in \mathbf{N}} \alpha(m, n)=\infty$.
Having in mind that

$$
\lim _{p \rightarrow \infty} a\left(e^{v p}\right)=\infty
$$

we find

$$
\sum_{p=0}^{\infty} a\left(e^{v p}\right)=\infty,
$$

which implies that there exists $k_{0} \in \mathbf{N}$ such that

$$
\sum_{p=0}^{k_{0}} a\left(e^{v p}\right) \geq C+1 .
$$

By our assumption and by condition i) it follows that

$$
\sup _{m \geq 0, n \geq k_{0}} \alpha(n, m)=\infty,
$$

and so there exist $m_{0}, n_{0} \in \mathbf{N}$, with $n_{0} \geq k_{0}$ and $\alpha_{m, n_{0}} \geq e^{w k_{0}}$. Now it is easy to check that

$$
\begin{aligned}
C & \geq \sum_{j=0}^{n_{0}} a\left(\frac{1}{e^{v j}} \alpha\left(n_{0}, m_{0}\right) \geq \sum_{j=0}^{n_{0}} a\left(\frac{1}{e^{v j}} e^{v k_{0}}\right)\right. \\
& \geq \sum_{j=0}^{k_{0}} a\left(e^{v\left(k_{0}-j\right)}\right)=\sum_{p=0}^{k_{0}} a\left(e^{v p}\right) \geq C+1,
\end{aligned}
$$

which is a contradiction. 


\section{The Main Result}

We start with the following

Lemma 3.1. If $a \in \mathcal{A}$ is a continuous convex function and if $T: \mathbf{N}^{2} \rightarrow \mathcal{B}(X)$ is an operator-valued function with the property that

$$
\sup _{m \in \mathbf{N}} \sum_{n=0}^{\infty} a(\|T(m, n) x\|)<\infty \quad(x \in X),
$$

then there exist $j_{0} \in \mathbf{N}, r_{0}>0$ such that

$$
\sup _{m \in \mathbf{N}} \sum_{n=0}^{\infty} a(\|T(m, n) x\|) \leq j_{0} \quad\left(x \in X \quad \text { with } \quad\|x\| \leq r_{0}\right) \text {. }
$$

Proof. For every natural number $j$ we consider the set

$$
H_{j}=\left\{x \in X: \sup _{m \in \mathbf{N}} \sum_{n=0}^{\infty} a(\|T(m, n) x\|) \leq j\right\} .
$$

From the fact that $a$ is continuous it follows that $H_{j}$ is a closed set and since $a$ is also convex it follows that $H_{j}$ is a convex set for all $j \in \mathbf{N}$. Using the hypothesis we can state that

$$
X=\bigcup_{j=0}^{\infty} H_{j}
$$

By Baire's theorem it follows that there exists $j_{0} \in \mathbf{N}$ such that $H_{j_{0}}$ has nonempty interior. Then there are $x_{0} \in X$ and $r_{0}>0$ such that every $y \in X$ with $\left\|y-x_{0}\right\| \leq r_{0}$ belongs to $H_{j_{0}}$. Let $x \in X$ with $\|x\| \leq r_{0}$ and $x_{1}=x+x_{0}, x_{2}=x-x_{0}$. Then $\left\|x_{1}-x_{0}\right\|=$ $\left\|-x_{2}-x_{0}\right\|=\|x\| \leq r_{0}$ and hence $x_{1},-x_{2}, x_{2} \in H_{j_{0}}$. Finally, by convexity of $H_{j_{0}}$ we obtain that

$$
x=\frac{1}{2} x_{1}+\frac{1}{2} x_{2} \in \frac{1}{2} H_{j_{0}}+\frac{1}{2} H_{j_{0}}=H_{j_{0}} .
$$

Now, we can state the main result of this paper.

Theorem 3.2. The evolution family $\mathcal{U}$ is u.e.d. if and only if there exist $a, b \in \mathcal{A}$ such that, for all $x \in X$,

$$
\begin{aligned}
\sup _{m \in \mathbf{N}} \sum_{k=0}^{\infty} a\left(\left\|U_{1}(k+m, m) P(m) x\right\|\right)<\infty \text { and } \\
\sup _{m \in \mathbf{N}} \sum_{k=0}^{m} b\left(\left\|U_{2}^{-1}(m, k) Q(m) x\right\|\right)<\infty .
\end{aligned}
$$


Proof. Necessity. It is a simple computation for $a(t)=b(t)=t$.

Sufficiency. Step 1. Let us define

$$
\alpha: \mathbf{N}^{2} \rightarrow \mathbf{R}_{+}, \alpha(n, m)=\frac{1}{M}\left\|U_{1}(n+m, m) Q(m) x\right\|
$$

where $x \in X$ is fixed arbitrary. It follows that

$$
\begin{aligned}
\sum_{j=0}^{n} a\left(\frac{1}{e^{w j}} \alpha(n, m)\right) & =\sum_{k=0}^{n} a\left(\frac{1}{M e^{w(n-k)}}\left\|U_{1}(n+m, m) P(m) x\right\|\right) \\
& \leq \sum_{k=0}^{n} a\left(\left\|U_{1}(k+m, m) P(m) x\right\|\right) \\
& \leq \sup _{m \in \mathbf{N}} \sum_{k=0}^{\infty} a\left(\left\|U_{1}(k+m, m) P(m) x\right\|\right)<\infty,
\end{aligned}
$$

for all $m, n \in \mathbf{N}$. By Lemma 2.7, it follows that $\sup _{m, n \in \mathbf{N}} \alpha(n, m)<\infty$, and hence by the principle of uniform boundedness we obtain that there exists $L_{1}>0$ such that, for all $m, n \in \mathbf{N}$,

$$
\left\|U_{1}(n+m, m) P(m)\right\| \leq L_{1} .
$$

Now it is easy to see that

$$
\begin{aligned}
\sup _{m \in \mathbf{N}} \sum_{k=0}^{\infty} A\left(\left\|U_{1}(k+m, m) P(m) x\right\|\right) & \\
& \leq L_{1}\|x\| \sup _{m \in \mathbf{N}} \sum_{k=0}^{\infty} a\left(\left\|U_{1}(k+m, m) P(m) x\right\|\right)<\infty,
\end{aligned}
$$

for all $x \in X$, where $A$ is the function defined in Remark 2.0, which belongs to $\mathcal{A}$ and is continuous and convex, and hence, by applying Lemma 3.1 to the operator-valued function $T: \mathbf{N}^{2} \rightarrow \mathcal{B}(X)$, $T(m, k)=U_{1}(k+m, m) P(m)$ it results that there exist $j_{1} \in \mathbf{N}$ and $r_{1}>0$ such that

$$
\sum_{k=0}^{\infty} A\left(\left\|U_{1}(k+m, m) P(m) x\right\|\right) \leq j_{1}
$$


for all $m \in \mathbf{N}$ and all $x \in X$ with $\|x\| \leq r_{1}$. A simple computation shows that

$$
\begin{aligned}
& \sum_{k=0}^{n} A\left(\left\|U_{1}(n+m, m) P(m) x\right\|\right)= \\
= & \sum_{k=0}^{n} A\left(\left\|U_{1}(n+m, m+k) P(m+k) U_{1}(m+k, m) P(m) x\right\|\right) \\
\leq & \sum_{k=0}^{n} A\left(L_{1}\left\|U_{1}(m+k, m) P(m) x\right\|\right) \\
= & \sum_{k=0}^{n} A\left(\left\|U_{1}(m+k, m) P(m)\left(L_{1} x\right)\right\|\right) \leq j
\end{aligned}
$$

for all $m, n \in \mathbf{N}$, and each $x \in X$ with $\|x\| \leq \frac{r_{1}}{L_{1}}$. Because A is also
bijective we have that

$$
\left\|U_{1}(n+m, m)\right\| \leq \frac{L_{1}}{r_{1}} A^{-1}\left(\frac{j_{1}}{n+1}\right) \quad(m, n \in \mathbf{N}) .
$$

From Lemma 2.6 it follows that there exist the constants $N_{1}, \nu_{1}>0$ such that

$$
\left\|U_{1}(t, s)\right\| \leq N_{1} e^{-\nu_{1}(t-s)} \quad(t \geq s \geq 0) .
$$

Step 2. Now fix $x \in X$ arbitrary and consider $\beta: \mathbf{N}^{2} \rightarrow \mathbf{R}_{+}$,

$$
\beta(n, m)=\frac{1}{M}\left\|U_{2}^{-1}(n+1, n) Q(n+1) x\right\| .
$$

Then we have

$$
\begin{array}{r}
\sum_{j=0}^{n} b\left(\frac{1}{e^{w j}} \beta(n, m)\right)=\sum_{k=0}^{n} b\left(\frac{1}{M e^{w(n-k)}}\left\|U_{2}^{-1}(n+1, n) Q(n+1) x\right\|\right) \\
=\sum_{k=0}^{n} b\left(\frac{1}{M e^{w(n-k)}}\left\|U_{2}(n, k) U_{2}^{-1}(n+1, k) Q(n+1) x\right\|\right)
\end{array}
$$




$$
\begin{aligned}
& \leq \sum_{k=0}^{n} b\left(\left\|U_{2}^{-1}(n+1, k) Q(n+1) x\right\|\right) \\
& \leq \sum_{k=0}^{n+1} b\left(\left\|U_{2}^{-1}(n+1, k) Q(n+1) x\right\|\right) \\
& \leq \sup _{l \in \mathbf{N}} \sum_{k=0}^{l} b\left(\left\|U_{2}^{-1}(l, k) Q(l) x\right\|\right)<\infty,
\end{aligned}
$$

for all $n, m \in \mathbf{N}$. As a consequence of Lemma 2.7. we obtain that

$$
\sup _{n \in \mathbf{N}}\left\|U_{2}^{-1}(n+1, n) Q(n+1) x\right\|<\infty \quad(x \in X),
$$

and by the principle of uniform boundedness it follows that

$$
\sup _{n \in \mathbf{N}}\left\|U_{2}^{-1}(n+1, n)\right\|<\infty .
$$

Now it is clear that there exists a constant $\delta>0$ such that

$$
\left\|U_{2}^{-1}(n, m)\right\| \leq e^{\delta(n-m)} \quad(n \geq m) .
$$

For $x$ an arbitrary vector of $X$, we define

$$
\gamma: \mathbf{N}^{2} \rightarrow \mathbf{R}_{+}, \gamma(n, m)=\left\|U_{2}^{-1}(n+m, m) Q(n+m) x\right\| .
$$

We have that, for all $m, n \in \mathbf{N}$,

$$
\begin{aligned}
& \sum_{j=0}^{n} b\left(\frac{1}{e^{\delta j}} \gamma(n, m)\right) \\
&=\sum_{j=0}^{n} b\left(\frac{1}{e^{\delta j}}\left\|U_{2}^{-1}(j+m, m) U_{2}^{-1}(n+m, j+m) Q(m+n) x\right\|\right) \\
& \leq \sum_{j=0}^{n} b\left(\left\|U_{2}^{-1}(n+m, j+m) Q(m+n) x\right\|\right) \\
& \leq \sum_{k=0}^{n+m} b\left(\left\|U_{2}^{-1}(n+m, k) Q(n+m) x\right\|\right) \\
& \leq \sup _{l \in \mathbf{N}} \sum_{k=0}^{l} b\left(\left\|U_{2}^{-1}(l, k) Q(l) x\right\|\right)<\infty .
\end{aligned}
$$

By applying once again Lemma 2.7 we have that $\sup _{n, m \in \mathbf{N}} \gamma(n, m)<\infty$, and hence by the principle of uniform boundedness we obtain that 
there exists $L_{2}>0$ such that, for all $n, m \in \mathbf{N}$,

$$
\left\|U_{2}^{-1}(n+m, m) Q(n+m)\right\| \leq L_{2} .
$$

Then it is easy to observe that

$$
\begin{aligned}
& \sup _{m \in \mathbf{N}} \sum_{k=0}^{m} B\left(\left\|U_{2}^{-1}(m, k) Q(m) x\right\|\right) \\
& \leq L_{2}\|x\| \sup _{m \in \mathbf{N}} \sum_{k=0}^{m} b\left(\left\|U_{2}^{-1}(m, k) Q(m) x\right\|\right)<\infty
\end{aligned}
$$

for all $x \in X$, where $B: \mathbf{R}_{+} \rightarrow \mathbf{R}_{+}, B(u)=\int_{0}^{u} b(s) d s$, which, by Remark 2.0, is continuous and convex. If we apply Lemma 3.1 to the operator-valued function $V: \mathbf{N}^{2} \rightarrow B(X)$ defined by

$$
V(n, m)= \begin{cases}U_{2}^{-1}(m, n) Q(m) & , \quad m \geq n \\ 0 & , \quad m<n\end{cases}
$$

we can state that there are $j_{2} \in \mathbf{N}, r_{2}>0$ such that

$$
\sup _{m \in \mathbf{N}} \sum_{k=0}^{m} B\left(\left\|U_{2}^{-1}(m, k) Q(m) x\right\|\right) \leq j_{2},
$$

for all $x \in X$ with $\|x\| \leq r_{2}$. It follows that

$$
\begin{aligned}
& \sum_{k=0}^{n} B\left(\left\|U_{2}^{-1}(m+n, m) Q(m+n) x\right\|\right) \\
&= \sum_{k=0}^{n} B\left(\| U_{2}^{-1}(k+m, m) Q(k+m) \times\right. \\
&\left.\quad \times U_{2}^{-1}(m+n, m+k) Q(m+n) x \|\right) \\
& \leq \sum_{k=0}^{n} B\left(L_{2}\left\|U_{2}^{-1}(m+n, k+m) Q(m+n) x\right\|\right) \\
&= \sum_{j=m}^{m+n} B\left(\left\|U_{2}^{-1}(n+m, j) Q(n+m)\left(L_{2} x\right)\right\|\right) \\
& \leq \sum_{j=0}^{n+m} B\left(\left\|U_{2}^{-1}(m+n, j) Q(m+n)\left(L_{2} x\right)\right\|\right) \leq j_{2},
\end{aligned}
$$


for all $m, n \in \mathbf{N}$, and all $x \in X$ with, $\|x\| \leq \frac{r_{2}}{L_{2}}$. Using the fact that $B$ is bijective too we obtain that, for all $m, n \in \mathbf{N}$,

$$
\left\|U_{2}^{-1}(n+m, m)\right\| \leq \frac{L_{2}}{r_{2}} B^{-1}\left(\frac{j_{2}}{n+1}\right) .
$$

In order to apply Lemma 2.6 we observe that

$$
U_{2}^{-1}\left(t, t_{0}\right)=U_{2}\left(t_{0},\left[t_{0}\right]\right) U_{2}^{-1}\left(\left[t_{0}\right]+2,\left[t_{0}\right]\right) U_{2}\left(\left[t_{0}\right]+2, t\right)
$$

for all $0 \leq t_{0} \leq t \leq t_{0}+1$ and hence

$$
\sup _{0 \leq t_{0} \leq t \leq t_{0}+1}\left\|U_{2}^{-1}\left(t, t_{0}\right)\right\| \leq M^{2} L_{2} e^{3 \omega} .
$$

Finally we obtain that there exists $N_{2}, \nu_{2}>0$ such that

$$
\left\|U_{2}^{-1}\left(t, t_{0}\right)\right\| \leq N_{2} e^{-\nu_{2}\left(t-t_{0}\right)} \quad, \quad \text { for all } t \geq t_{0} \geq 0 .
$$

The necessity of Theorem 3.2 is not true for all $a, b \in \mathcal{A}$ as the following example illustrates.

Example 3.3. Let $X=\mathbf{R}, U(t, s)=e^{-(t-s)}, P(t)=1, a(u)=$ $\sum_{n=1}^{\infty} \frac{\sqrt[n]{u}}{n^{2}}$. It is clear that $\mathcal{U}=\{U(t, s)\}_{t \geq s \geq 0}$ is u.e.d. but for $x=1$, we have

$$
\begin{aligned}
\sum_{k=0}^{\infty} a\left(\left\|U_{1}(k+m, m) P(m) x\right\|\right) & =\sum_{k=0}^{\infty} a\left(e^{-k}\right)=\sum_{k=0}^{\infty} \sum_{n=1}^{\infty} \frac{1}{n^{2}} e^{-\frac{k}{n}} \\
& =\sum_{n=1}^{\infty} \sum_{k=0}^{\infty} \frac{1}{n^{2}} e^{-\frac{k}{n}} \\
& =\sum_{n=1}^{\infty} \frac{1}{n^{2}} \frac{e^{\frac{1}{n}}}{e^{\frac{1}{n}}-1}=\infty,
\end{aligned}
$$

for all $m \in \mathbf{N}$.

Theorem 3.4. The evolution family $\mathcal{U}$ is u.e.d. if and only if there exist $K, L, p, q>0$ such that

$$
\begin{aligned}
& \sum_{n=m}^{\infty}\|U(n, m) x\|^{p} \leq K\|x\|^{p} \quad(m \in \mathbf{N}, x \in \operatorname{ImP}(m)) \quad \text { and } \\
& \sum_{n=m}^{l}\|U(n, m) x\|^{q} \leq L\|U(l, m) x\|^{q} \quad(m \geq l, x \in \operatorname{Ker} P(m)) .
\end{aligned}
$$

Proof. Follows easily from Theorem 3.2 for $a(u)=u^{p}, b(u)=u^{q}$. 


\section{REFERENCES}

[1] C. Chicone, Y. Latushkin, Evolution semigroups in dynamical systems and differential equations, Mathematical Surveys and Monographs 70, American Mathematical Soxiety, Providence, RI, 1999.

[2] R. Datko, Extending a theorem of Liapunov to Hilbert spaces, J. Math. Anal. Appl. 32 (1970), 610 - 616.

[3] R. Datko, Uniform asymptotic stability of evolutionary processes in a Banach space, SIAM J. Math. Analysis 3 (1973), 428 - 445.

[4] D. Henry, Geometric theory of semi-linear parabolic equations, SpringerVerlag, New York, 1981.

[5] J. P. La Salle, The stability and control of discrete processes, SpringerVerlag, Berlin, 1990.

[6] W. Littman, A generalization of the theorem Datko-Pazy, Lecture Notes in Control and Inform. Sci. 130 (1983), 318 - 323.

[7] M. Megan, A. Pogan, On a theorem of Rolewicz for semigroups of operators in locally convex spaces, Ann. Math. Blaise Pascal 7 (2000), 23 - 35.

[8] J. M. A. M. van Neerven, Exponential stability of operators and semigroups, J. Func. Anal. 130 (1995), 293 - 309.

[9] J. M. A. M. van Neerven, The asymptotic behavior of semigroups of linear operators, Theory, Advances and Applications 88, Birkhäuser, 1996.

[10] A. Pazy, On the applicability of Liapunov's theorem in Hilbert spaces, SIAM J. Math. Anal. Appl. 3 (1972), 291 - 294.

[12] A. Pazy, Semigroups of linear operators and applications to partial differential equations, Springer-Verlag, 1983.

[13] M. Pinto, Discrete dichotomies, Computers Math. Appl. 28 (1994), 259 270.

[14] P. Preda, M. Megan, Exponential dichotomy of evolutionary processes in Banach spaces, Czech. Math. J. 35 (1985), 312 - 323.

[15] K. M. Przyluski, S. Rolewicz, On stability of linear time-varying infinitedimensional discrete-time systems, Systems Control Lett. 4 (1994), 307 315.

[16] S. Rolewicz, On uniform N-equastabily, J. Math. Anal. Appl. 115 (1986), $434-441$.

[17] J. Zabczyk, Remarks on the control of discrete-time distributed parameter systems, SIAM J. Control. Optim. 12 (1974), 721 - 735.

[18] Q. Zheng, The exponential stability and the perturbation problem of linear evolution systems in Banach spaces, J. Sichuan Univ. 25 (1988), 401 - 411.

Department of Mathematics,

West University of Timişoara,

Bd. V. Pârvan, no. 4,

1900-Timişoara, Romania

preda@math.uvt.ro, ciprian.preda@fse.uvt.ro

Received on 1 April 2003 and in revised form on 14 January 2004. 